\title{
Evaluación de la calidad del servicio de transporte urbano colectivo intramunicipal por los usuarios de la ciudad de Estelí, año 2016 ${ }^{1}$
}

\author{
Aleyda Lourdes Benavidez Vásquez ${ }^{2}$ \\ Josué Enmanuel Mendoza Castillo ${ }^{3}$ \\ Lesther Agustín Zelaya López ${ }^{4}$ \\ Beverly Castillo Herrera ${ }^{5}$
}

\section{RESUMEN}

El presente estudio Evalúa la calidad del servicio de transporte urbano colectivo intramunicipal en la ciudad de Estelí para la satisfacción de los usuarios en el año 2016. El servicio presenta deficiencias para los usuarios en función de la calidad física de las unidades (puertas abiertas, falta de higiene, asientos deteriorados) y de la calidad de la atención a los usuarios (exceso de pasajeros, trato inadecuado, inseguridad, violencia).Esta investigación es de tipo cuantitativa con un universo de estudio de 379 usuarios del transporte, 31 conductores y 58 ayudantes. Se utilizaron encuestas, entrevistas y guías de observación como herramientas de evaluación. La hipótesis de la investigación se basó en que la baja calidad del servicio de transporte urbano colectivo intramunicipal incide en la satisfacción del usuario, además se hace énfasis en las debilidades más urgentes expuestas por los usuarios así como de las vulnerabilidades del servicio y sus faltas más frecuentes tales como la poca o nula supervisión por parte de las autoridades. Se sugiere que se implementen estrategias de mejora como un método más moderno de cobro por ejemplo cobro electrónico además de mejorar las condiciones de las paradas de los autobuses y demás mejoras estéticas del servicio, los principales resultados obtenidos con este estudio es que a pesar de las notorias deficiencias del servicio hay una alto margen de aceptación por parte de los usuarios en cuanto a la calidad del servicio, ya que en comparación con el servicio de otras ciudades, Estelí cuenta con uno de los mejores.

Palabras clave: Transporte, calidad, urbano, intramunicipal, Estelí.

Recibido: 12 de diciembre de 2016

Aceptado: 16 de febrero de 2017

1 Este artículo se basa en la investigación titulada Evaluación de la calidad del servicio de transporte urbano colectivo intramunicipal por los usuarios de la Ciudad de Estelí año 2016. Para optar al título de Licenciatura en Administración de Empresas por la UNAN-Managua, FAREM-Estelí.

2 UNAN-Managua, FAREM-Estelí. Correo electrónico: adyelalove@gmail.com

3 UNAN-Managua, FAREM-Estelí. Correo electrónico:mjosue2709@gmail.com

4 UNAN-Managua, FAREM-Estelí. Correo electrónico:lesterzelaya21@gmail.com

5 Doctora en Ciencias Sociales por la Universidad del Zulia, Venezuela. Maestría en Ciencias Sociales por la Universidad de Guadalajara, México. Docente titular de la UNAN-Managua, FAREM-Estelí, Correo electrónico: beverly.castillo@yahoo.com 


\title{
Evaluation of the quality of intra-municipal collective urban transport service by users of the city of Estelí, year 2016
}

\begin{abstract}
This study evaluates the quality of intramunicipal urban public transport in the city of Estelí to the satisfaction of users in 2016. The service is deficient for users based on the physical quality of the units (open doors, unhygienic, damaged seats) and the quality of care users (excess passengers, inadequate treatment, insecurity and violence). This research is quantitative type with a universe of study of 379 transport users, 31 drivers and 58 assistants. For this investigation we use surveys, interview and observation guides as assessment tools. The hypothesis of the research was based on the poor service quality of urban public transport, its most frequent absences such as little or no supervision by the authorities. It is suggested that improvement strategies as a more modern method of collection is implemented, for example electronic billing, in addition to improving the conditions of bus stops and also improvements esthetic service, the main results obtained from this study is that despite the glaring deficiencies of the service there is a high margin of acceptance by users in terms of service quality, as compared to the service of other cities, Estelí has one of the best.
\end{abstract}

Keywords: Transport, quality, urban, intramunicipal, Estelí. 


\section{INTRODUCCIÓN}

En el año 2016 se ha visto más afectada la satisfacción de los usuarios del transporte urbano colectivo intramunicipal en la ciudad de Estelí generando una problemática más, mediante este estudio se pretende también concientizar a los dueños de las unidades, trabajadores de las mismas e incluso a las autoridades correspondientes a mejorar el servicio.

La inseguridad en las unidades es un sentir generalizado en los usuarios, hay algunos barrios catalogados como peligrosos y ahí transitan ciertas rutas; como por ejemplo la ruta del Oscar Gámez, Robacasa y Sandino, por lo tanto es inevitable que las unidades sean abordadas por delincuentes y antisociales, quienes se aprovechan cuando las unidades viajan saturadas para cometer actos ilícitos.

El maltrato hacia los usuarios por los conductores y ayudantes, por el lenguaje vulgar y grosero que utilizan, además de conductas inapropiadas hacia las mujeres que abordan la unidad e incluso el exceso de volumen del radio dentro de la ruta perturba la tranquilidad del usuario durante el recorrido hacia su destino.

El exceso de pasajeros en las rutas urbanas genera incomodidad a la hora de abordar la unidad de tal manera que no hay espacio suficiente sobre todo en horas de mayor afluencia, provocando golpes, lesiones y daños a sus pertenencias.

También es evidente el incumplimiento de las medidas de higiene dentro de las unidades, puesto que no todos cuentan con un depósito de basura, para que los usuarios puedan depositar la misma.

El transporte público colectivo intramunicipal es un servicio regulado por las Alcaldías Municipales y el Ministerio de Transporte e Infraestructura; cuyos fundamentos están dentro del marco jurídico de la Ley 524, Ley General de Transporte Terrestre.
Este estudio servirá para que los propietarios de las unidades ofrezcan mejores condiciones del servicio, con seguridad, higiene y comodidad a los usuarios, además, exigiendo a los conductores que cumplan estrictamente con las leyes de transito garantizando de manera tal, el orden público reduciendo los accidentes y logrando la satisfacción del usuario.

Esta investigación representa grandes beneficios para el sector en estudio puesto que se pretende detectar las debilidades en la prestación del servicio y proponer estrategias que contribuyan a su mejoramiento; que generen mayor satisfacción en los usuarios que utilizan este servicio. Se pretende evaluar la calidad del servicio del transporte urbano colectivo intramunicipal en la ciudad de Estelí para la satisfacción de los usuarios.

El transporte público urbano puede ser proporcionado por una o varias empresas privadas, por cooperativas o consorcios de transporte público. Los servicios se mantienen mediante cobro directo a los pasajeros. Normalmente son servicios regulados y subvencionados por autoridades locales o nacionales. Este transporte

es el servicio público de pasaje que se presta en el interior de una ciudad o pueblo mediante rutas previamente establecidas en autobuses o camiones a cambio del pago de una tarifa que normalmente fija el gobierno. (Satillana , 2007)

\section{MATERIALES Y MÉTODOS}

En la primera etapa de la investigacion se recopilo informacion secundaria sobre el tema, para diseñar el protocolo de la ivestigacion. Se consultaron libros de administracion de empresa y mercadotecnia sobre atencion al cliente, calidad y municipio en la Biblioteca Urania Zelaya de la FAREM'Estelí. Ademas se cosultaron articulos en linea y paginas web para dar veracidad a la informacion de la investigacion. 
Esta es una investigación aplicada dado que percibe fines inmediatos para dar respuesta positiva hacia la resolución de una problemática y según el enfoque filosófico; es una investigación cuantitativa analítica.

El universo de estudio fueron 34 unidades del transporte urbano, de lo cual se conocía que el promedio diario de personas que utilizan el servicio es de 27,200 usuarios. Además se aplicó la fórmula para calcular la muestra de la población finita obteniendo una resultado de 379 usuarios, con la misma manera se calculó el muestreo para los conductores y ayudantes de las unidades.

Se encuestaron 31 conductores y 58 ayudantes distribuidos en los diferentes barrios de la Ciudad de Estelí, además, también se entrevistó al Director del Transporte General de la Alcaldía Orlando Castillo, para obtener información necesaria para la investigación.

Además se elaboraron diferentes instrumentos que ayudaron a la obtención de información real tales como: entrevistas, encuestas y guías de observación, por ultimo para el procesamiento de dicha información se utilizó la herramienta de IBM SPSS.

\section{FUNCIONAMIENTO}

Los propietarios de las unidades para con los ayudantes deben cumplir con parámetros establecidos por la Ley General de Transporte, los cuales deben estar en óptimas condiciones físicas y de salud, por otra parte los trabajadores de las unidades reciben anualmente capacitaciones contra cualquier situación de riesgo que se presente a un usuario en caso de emergencia.

\section{RESULTADOS Y DISCUSIÓN}

\section{Descripción breve}

El transporte urbano intramunicipal de la ciudad de Estelí ha sido uno de los servicios más usados por los ciudadanos de este municipio prácticamente desde que fue necesario su implementación casi tras que fue elevada a ciudad y a medida que se ha ido expandiendo territorialmente y también con su constante explosión demográfica ya que cada vez es más lejos la distancia que los ciudadanos deben de recorrer para movilizarse a sus puntos más frecuentes como por ejemplo lugares de estudio, centros de trabajo, etc.

Sin embargo con el crecimiento exponencial de la demanda del servicio se presentan ciertos inconvenientes tales como la mala calidad del mismo muchas veces debido a la poca o nula supervisión y control por parte de las autoridades correspondientes generando problemas habituales y cotidianos.

Por tanto se deben cumplir con ciertos parametros que exige la ley 524, Ley general de transporte terrestre, por lo tanto se debe tener claro el concepto que abarca calidad en un producto o servicio.

(Reyes, 2011) Calidad es cuando se logra un producto es económico, útil y satisfactorio para el consumidor Se dice que un producto o un servicio es de calidad cuando satisface las necesidades o expectativas del usuario o cliente, en función de parámetros como:

- Seguridad que el producto o servicio confieren al cliente.

- Fiabilidad o capacidad que tiene el producto o servicio para cumplir las funciones especificadas, sin fallos y por un periodo determinado.

- Servicio o medida que el fabricante y distribuidor responden en caso de fallo del producto o servicio.

\section{Organización}

Se puede observar como los lugares más alejados del centro de la ciudad son los que cuentan con menos unidades de buses que cubran la demanda del servicio en esas zonas siendo ese uno más de los problemas mas notable. 
De ese total de 34 unidades de transporte urbano colectivo intramunicipal, está estructurado en cinco rutas y tres distritos de los cuales están distribuidas de la siguiente manera:

- Ruta número uno; inicia su recorrido en la Rotonda El Rosario contiguo a FAREM-Estelí, recorriendo el Barrio El Rosario y finalizando en el Hospital San Juan de Dios. El recorrido tiene una duración de punto de salida, a punto de llegada de 45 minutos exactos, el cual debe cumplirse, en caso contrario se impone una sanción. Dicha ruta cuenta con nueve unidades independientes, es decir, no existe cooperativa que las una o sindicalice; y se tiene establecido un intervalo de 10 minutos que indica el tiempo de salida entre una unidad y otra.

- Ruta número dos; saliendo de Rotonda El Rosario contiguo a FAREM-Estelí, recorriendo el Barrio Oscar Gámez y finalizando en el Hospital San Juan de Dios. Cuenta con nueve unidades independientes y se tiene establecido un intervalo de 10 minutos que indica el tiempo de salida entre una unidad y otra.

- Ruta número tres; saliendo del Barrio Sandino y finalizando en el Hospital San Juan de Dios. Esta ruta cuenta con seis unidades, y se tiene establecido un intervalo de 12 minutos que indica el tiempo de salida entre una unidad y otra.

- Ruta número cuatro; saliendo de Villa Cuba y finalizando en el Hospital San Juan de Dios. Esta ruta cuenta con cuatro unidades, y se tiene establecido un intervalo de 18 minutos que indica el tiempo de salida entre una unidad y otra.

- Ruta número cinco; iniciando en Roba Casa (carretera a La Concordia), desviándose a la UNIRUACS para luego dirigirse hacia el Hospital San Juan de Dios y se tiene establecido un intervalo de 18 minutos que indica el tiempo de salida entre una unidad y otra.

- Ruta número seis; iniciando su salida desde la universidad Campus Medico UCATSE seguido del barrio La. Thompson, finalizando su recorrido Hospital.
A continuación se presenta el cuadro de distribución de las unidades:

\section{Cuadro $N^{\circ} 1$. Distribución de unidades}

\begin{tabular}{lll}
\hline$N^{\circ}$ & Cantidad rutas & Salida/Entrada \\
\hline 1 & 9 unidades & Rosario/Hospital \\
2 & 9 unidades & Oscar Gámez/Hospital \\
3 & 6 unidades & $\begin{array}{l}\text { Sandino/Hogar chavalito/ } \\
\text { Hospital }\end{array}$ \\
& & Roba Casa/Hospital/UNI \\
4 & 4 unidades & Villa cuba/Hospital \\
5 & 4 unidades & El Dorado/Hopital \\
6 & 2 unidades & \\
\hline Total & 34 unidades & \\
\hline
\end{tabular}

Entre las cosas que si tiene a favor el servicio según los usuarios es la buena puntualidad además del buen estado mecánico de las unidades de las rutas de los barrios del Rosario y Oscar Gámez hacia el hospital; también la tarifa es bastante aceptada por la población en general y el trayecto de cada recorrido es favorable según los puntos clave de la ciudad y se cuenta con las suficientes unidades para que el intervalo de tiempo que hay entre una y otra sea lo mas breve posible.

Hay muchas maneras de en las que se podría aprovechar mas el servicio de transporte urbano colectivo intramunicipal por ejemplo a manera estratégica meter rutas emergentes los días domingos y feriados ya que esos días es poco el flujo de unidades, sin embargo, la población sigue necesitando del servicio, además se podría promocionar y aumentar el turismo mediante un mejor uso del servicio e incluso se pueden aumentar las concesiones debido al crecimiento poblacional que se ha registrado de manera exponencial los últimos años situacion que ha generado nuevas calles e incluso nuevos barrios; en negociaciones mas apropiadas y oportunas con el gobierno se podrían efectuar arreglos a manera de subsidios sobre el combustible, repuestos e incluso unidades nuevas para el servicio.

Asi mismo también hay cosas que evidentemente se deben de mejorar, entre ellas destacan las siguientes: puertas abiertas de las unidades durante el trayecto, 
que los trabajadores de las mismas no usan uniformes característicos o vestimentas apropiadas para la labor, el exceso de pasajeros que abordan las unidades sobre todo en las horas de mayor afluencia y dando como resultado un desagradable recorrido al usuario traducido o fluctuado en un trato inadecuado a los mismos por parte de los colaboradores de la unidades, se observa también la falta de higiene dentro de los buses y el deterioro de los asientos; todo esto es la mayor problemática que posee actualmente el servicio de transporte urbano intramunicipal en la ciudad de Estelí.

Ademas se presentan muchos inconvenientes, algunos que incluso no dependen de los transportistas, tal es el caso cuando celebran actos populares en las calles $\mathrm{y}$ avenidas principales de la ciudad lo que obliga a los conductores a tomar rutas alternas de manera que se hace casi imposible poder terminar en horario y forma correcto sus recorridos, también pueden darse estos atrasos debido a cortejos fúnebres o reparación de calles de manera aleatoria y sin previo aviso a los transportistas.

La escasa afluencia de usuarios los días lluviosos a causa de falta de casetas en las paradas que presten condiciones optimas para que las personas puedan esperar de manera tranquila y sin afectaciones a que pasen las unidades de buses por lo tanto las personas optan mas por el uso de taxis para poder movilizarse; la saturación del parque vehicular y las pequeñas calles de esta ciudad también son un impedimento de gran importancia sobre todo que la falta de control por parte de las unidades evita que se resuelva esta problemática sumado a la falta de cultura social son los elementos mas destacables que generan estas inconformidades.

\section{Requisitos de concesión}

Para que una persona natural o jurídica brinde el servicio de transporte debe ser propietario de una concesión de ruta, esta se consigue mediante una licitación al momento de abrirse una nueva ruta o de caducar la anterior, la vigencia de la concesión es de 20 años, durante esos 20 años se puede pasar a un tercero, ya sea por venta o por cesión, sin embargo este tercero debe pagar a la municipalidad 14,000 córdobas para que la alcaldía pase todo a nombre del nuevo propietario con su nueva y debida documentación.

Para generar el ingreso de una nueva unidad de transporte a este sector, se debe cumplir con una serie de requisitos, siendo el de mayor relevancia la demanda de esta misma; lo cual debe ser presentado de manera formal, a través de un estudio técnico del crecimiento poblacional, dirigido a la población económicamente activa (PEA). Antes el estudio técnico lo realizaba el interesado en obtener dicha ruta; pero en la actualidad se debe hacer una solicitud formal a la Alcaldía Municipal quien se encarga de ejecutar dicho estudio y partiendo de los resultados que este arroje se aprueba o se rechaza la solicitud.

La base mínima para dar una concesión es de 50 mil córdobas, hay tazas de pagos fijos, por ejemplo cuando alguien gana la licitación tiene 3 días tope para pagar la oferta, 500 córdobas como impuesto anual, además el propietario debe pagar 300 córdobas mensuales por brindar dicho servicio, sin embargo, hay otros parámetro. Orlando Castillo expresa:

"Como Alcaldía debemos garantizar la calidad del servicio de transporte publico intramunicipal, por lo tanto al evaluar los diferentes tipos de opciones de concesiones, se debe tomar en cuenta las óptimas condiciones de la unidad como por ejemplo: Año de urbano, estado mecánico, comodidad para el usuario; aunque el mínimo para la concesión sea de C\$ 50,000.00 pueden existir otras opciones más altas en cuanto a la concesión, por ejemplo el año del bus, se elige el más favorable y que cumpla con las necesidades del usuario".

Los usuarios participantes del estudio, opinan con un $56.9 \%$ que la puntualidad del servicio de transporte 
urbano es muy buena, el $34.4 \%$ la consideran regular y el $8.6 \%$ restante califican que es mala.

Sobre la puntualidad Orlando Castillo, Director de Transporte, Alcaldía expresa:

"La puntualidad en el servicio público es fundamental, se basan en un horario establecido de entrada, salida, y parada. La alcaldía cuenta con chequeadores que se encargan de estar al pendiente de los horarios en los que debe pasar una ruta por un punto establecido; si los conductores no cumplen con el horario establecido o no llegan a tiempo se les asigna una multa por ir colgados con el tiempo, salvo que por algún motivo ajeno a las circunstancias por ejemplo que ENACAL este reparando calles, Unión Fenosa repare un poste, por congestionamiento vehicular entre muchas otras causas, se retrase el conductor debe avisar al chequeador que va retrasado sino se aplica la multa".

Es fundamental que los conductores cumplan con la puntualidad del servicio dado que la mayoría de los usuarios que utilizan el transporte tienen conocimiento de la hora que pasa cada unidad y si la unidades son impuntuales genera inconvenientes, retrasos, en las actividades cotidianas de los usuarios provocando insatisfacción de la calidad del servicio, sin embargo, en Estelí el horario se cumple de manera eficiente y así los expresan los usuarios generando una aceptación de más de la mitad de personas que ayudaron al estudio recalcando que la mayoría de las unidades cumplen con los horarios establecidos por la Alcaldía.

\section{Condiciones físicas, psicológicas y de seguridad.}

El 54.4\% de los usuarios participantes del estudio del servicio de transporte urbano colectivo intramunicipal opinan que la higiene de las unidades es regular, el $27.3 \%$ la consideran buena, y el restante $18.3 \%$ mala.

Según el mercadólogo mexicano Muñiz, J (2011) que tiene experiencia en medir la satisfacción de los clientes define el concepto de satisfacción como: " $E l$ nivel del estado de ánimo de una persona que resulta de comparar el rendimiento percibido de un producto o servicio con sus expectativas".

Por tanto es importante para los usuarios que utilizan el transporte urbano colectivo intramunicipal que se cumplan los requisitos que exige la alcaldia, de acuerdo a la higiene dentro de las unidades gran parte de la poblacion encuestada califico como regular, razon por la cual no se sienten satisfechos con este elemento indispensable para la calidad del servicio. Este tipo de normativas estan establecidas en la ley 524, Ley General de transporte terrestre y Ley de Municipalidades, por tal razon el propietario en conjunto con los colaboradores de las unidades deberan cumplir sino se les asignara una multa establecida en la presente ley.

El 50\% de los usuarios encuestados opina que la comodidad de las unidades de transporte urbano colectivo intramunicipal es regular, el $33 \%$ afima que es buena y el $17 \%$ expresa su inconformidad con la comodidad que brindan los propietarios de la sunidades de transporte, resumido de la siguiente manera:

\section{Cuadro $\mathrm{N}^{\circ} 2$. Comodidad de las unidades}

\begin{tabular}{lrrrr}
\hline & Buena & Regular & Mala & \multicolumn{1}{c}{ Total } \\
\hline Frecuencia & 126 & 187 & 66 & 379 \\
Porcentaje & $33 \%$ & $50 \%$ & $17 \%$ & $100 \%$ \\
\hline
\end{tabular}

Fuente: Datos primarios encuesta a usuarios, Junio 2016

Calidad se define: Es la totalidad de los rasgos y características de un producto o servicio que se sustenta en su habilidad para satisfacer las necesidades y expectativas del cliente, y cumplir con las especificaciones con la que fue diseñado.

Es importante que los elementos de la calidad se cumplan de manera eficiente para la satisfacción de los usuarios que utilizan el servicio de transporte urbano colectivo intramunicipal. Existen muchos factores que conforman la comodidad dentro de la prestación del servicio de transporte, y este es fundamental para 
las y los usuarios viajen en un ambiente agradable, confortable, a sus destinos, sin embargo existe un mayor porcentaje de personas que no están satisfechas dado que catalogan este elemento como regular; dejando aspectos negativos que no logren calificarla como buena.

E1 66.4\% de los usuarios participantes del estudio de la evalucion de la calidad del servicio de transporte opinan que los agarraderos si estan a la altura adecuada por tanto el $33.9 \%$ restante considera que no estan a la altura adecuada.

Según Muñiz,(2011) define comodidad: hace referencia a la comodidad que se le brinda al cliente cuando visita el local. Se da, por ejemplo, cuando el local cuenta con espacios lo suficientemente amplios como para que el cliente se sienta a gusto, sillas o sillones cómodos, mesas amplias, elementos primordiales en la prestación de servicios, estacionamiento vehicular, un lugar en donde pueda guardar sus pertenencias, etc.

Cabe mencionar que la comodidad es un factor importante en la satisfacción de los usuarios, los usuarios buscan como sentirse cómodos en una unidad de transporte, sin embargo, estos parámetros deben cumplirse por parte de los propietarios de las unidades; a mayor comodidad mayor satisfacción por parte de la gente. La ley 524 hace referencia a los requisitos que deben cumplir para brindar un mejor servicio y aunque los agarraderos son de una altura estándar muchos niños, ancianos, dicen quejarse por la altura de estos porque son muy altos en algunas unidades. Por tanto los propietarios deben generar soluciones para lograr la aceptación de la población que utiliza el servicio, cuando son muy altos no existe la posibilidad de agarrarse en los pasamanos.

Parte fundamental de cualquier negocio es la atención que le dan a los usuarios, de la muestra que se tomó para encuestarse el $27.2 \%$ se siente completamente satisfecho con la atención que recibe al momento de usar el servicio de transporte mientras que la mayoría no lo considera muy mal pero tampoco muy bien, es decir, quedándose en un rango medio siendo estos el $51.4 \%$ de los encuestados, sin embargo, el $21.4 \%$ no se siente para nada satisfecho con la atención recibida.

\section{Cuadro $\mathrm{N}^{\circ}$ 3. Atención a los usuarios}

\begin{tabular}{lrrrr}
\hline & Muy buena & Regular & Mala & Total \\
\hline Frecuencia & 106 & 190 & 83 & 379 \\
Porcentaje & $27.2 \%$ & $51.4 \%$ & $21.4 \%$ & $100 \%$ \\
\hline
\end{tabular}

Fuente: Datos primarios encuesta a usuarios, Junio 2016

El servicio al cliente es el conjunto de estrategias que una compañía diseña para satisfacer, mejor que sus competidores, las necesidades y expectativas de sus clientes externos siendo la atención la piedra angular de dicho conjunto" (Serna, 2006)

A pesar de que es una leve minoría las personas que no se sienten para nada conformes con la atención que reciben al usar el servicio, hay realmente mucho malestar si a eso le sumamos las personas que consideran regular la atención de manera que es un factor a tomarse muy en cuenta respecto a las estrategias que se pueden implementar para mejorar el servicio.

De manera muy similar que con la atención en el servicio también el trato que el usuario recibe de parte de los trabajadores de la unidad es de calidad media ya que una gran cantidad de las personas encuestadas considera regular el trato siendo estas el $49.7 \%$, solo un $33.1 \%$ lo considera muy bueno el trato y un $17.2 \%$ le parece malo del todo.

Figueroa, E. (2009) El mayor porcentaje de pérdida de clientes y usuarios es por haberse sentido despreciados por alguien del personal, bien por una mala atención o por indiferencia. Solo es necesario causar una vez esta sensación en el para que se aleje para siempre.

Tomando en cuenta este concepto vemos lo fácil que es perder la clientela incluso a veces de manera no 
intencional, por lo tanto, es de suma importancia que los trabajadores de las unidades tengan conocimientos básicos al menos sobre atención al cliente.

Algunas de las cosas que le pueden quitar la tranquilidad a un usuario son los conflictos que puede tener o incluso presenciar dentro de las unidades, al efectuarse el estudio se encontró que el $78.6 \%$ si ha pasado por este inconveniente mientras que el $21.4 \%$ ha conseguido evitarlos.

Figueroa, E. (2009) Un cliente perdonara un error si se le atiende correctamente y se soluciona de forma eficaz pero es muy difícil que olvide una agresión por parte del personal y mucho menos, si siente que se le ha tratado mal o como un objeto.

Es necesario, por tanto, disponer del personal adecuado para atender a los usuarios o clientes ya que esto garantizara su satisfacción y aumentara su confianza en la empresa.

Otra de las cosas que afectan la condición psicológica del usuario al abordar las unidades de transporte es el inadecuado volumen del estéreo de las unidades, el $47.5 \%$ de los usuarios encuestados consideran normal el volumen del estéreo, un $24.2 \%$ lo considera bajo y un $28.3 \%$ le parece ruidoso y de mal gusto.

Muñiz (2011) define ambiente agradable como: es un ambiente acogedor en donde el cliente se siente a gusto. Se da, por ejemplo, cuando los trabajadores le dan al cliente un trato amable y amigable, cuando el local del negocio cuenta con una buena decoración, una iluminación adecuada, una música agradable, etc.

De esta manera podemos observar que hasta los detalles más pequeños tiene un magnitud significativa cuando hablamos de la satisfacción del cliente, cosas tan pequeñas como la limpieza de la unidad o el volumen del radio pueden hacer una diferencia importante en el usuario.
El $52.5 \%$ de los usuarios encuestados afirmaron estar satisfechos con la seguridad que brindan los conductores, mientras el $47.5 \%$ dijo haber estado inconforme con el servicio que brindan los responsables de las unidades que conducen.

Ésta hace referencia a la seguridad que existe en el local y que, por tanto, se le da al cliente al momento de visitarlo. Se da, por ejemplo, cuando se cuenta con suficiente personal de seguridad para evitar que los delincuentes se aprovechen de los usuarios, cuando se tienen claramente marcadas las zonas de seguridad, cuando se tienen claramente señalizadas las vías de escape, cuando se cuenta con botiquines médicos, cuando hay un plan de emergencia a la hora de un accidente.

Este estudio nos refleja que la mayoría de los usuarios no están satisfechos con la seguridad que brindan los dueños y las unidades transporte, ya que hay factores que inciden en el riesgo de la seguridad que estos mismos ofrecen y deben mejorar, a través de este estudio se determinaron causas las cuales afectan el servicio y el desempeño máximo de las unidades, para que esto suceda los propietarios deben garantizar una mejor calidad de servicio.

Tambien el 71.1\% de los usuarios califican de manera prudente la forma de conducir de los choferes de las unidades, el $22.2 \%$ refiere que la manera de este es imprudente, mientras que el restante $6.7 \%$ dicen, que conducen de una manera agresiva poniendo en riesgo la seguridad de los usuario.

Según el artículo 9 de la ley 524, Ley General de Trasporte expresa que, todos los conductores y el personal auxiliar del servicio público de transporte terrestre deberán someterse periódicamente a los exámenes médicos correspondientes, los que deberán hacer énfasis en detectar el uso de drogas o sustancias que pongan en peligro la vida y los bienes de los usuarios, todo de conformidad con lo establecido en la 
presente Ley, en la Ley para el Régimen de Circulación Vehicular e Infracciones de Tránsito y a la normativa que elabore el MTI para tal efecto.

En base a los resultados obtenidos, reflejala conformidad de los usuarios a la hora de conducir ya que de manera prudente circulan por su recorrido brindando la seguridad que la mayoría de los usuarios demandan, sin embargo hay errores que los conductores cometen a la hora de conducir poniendo en riesgo la vida del demandante del servicio, esta parte de la población dice estar en desacuerdo y exclaman que necesitan más seguridad y conductores más capacitados para esta labor.

Dentro de las principales causas de inseguridad que se presentan en las unidades de transporte urbano colectivo, los usuarios de las unidades respondieron; que la mayor causa de inseguridad con un $33.3 \%$ representa el exceso de pasajeros, el $26.4 \%$ de la delincuencia que se presentan en las unidades, por otra parte el $22.8 \%$ circulan con las puertas abiertas siendo un peligro para los usuarios, y no obviando las condiciones mecánicas de la unidad con un 2.8\% mientras que el $14.7 \%$ respondió que todas las causas anteriores se vivían en el transcurso de la ruta. Los concesionarios están obligados a cumplir con las normas y técnicas, a fin de garantizar la seguridad y comodidad de los usuarios y la protección del medio ambiente; todos los vehículos de servicio público terrestre, deberán cumplir con normas y técnicas para la inspección de sus condiciones físicas y mecánicas, y obtener la constancia de aprobación correspondiente con la periodicidad y términos que establezca esta Ley y su Reglamento.

\section{Cuadro $N^{\circ}$ 4. Seguridad de los pasajeros}

\begin{tabular}{lrrrrrr}
\hline & Delincuencia & \multicolumn{1}{c}{$\begin{array}{c}\text { Exceso de } \\
\text { pasajeros }\end{array}$} & \multicolumn{1}{c}{$\begin{array}{c}\text { Puertas } \\
\text { abiertas }\end{array}$} & \multicolumn{2}{c}{$\begin{array}{c}\text { Condiciones } \\
\text { mecánicas }\end{array}$} & \multicolumn{2}{c}{$\begin{array}{c}\text { Todas las } \\
\text { anteriores }\end{array}$} & Total \\
\hline Frecuencia & 95 & 125 & 87 & 14 & 58 & 379 \\
Porcentaje & $26.4 \%$ & $33.3 \%$ & $22.8 \%$ & $2.8 \%$ & $14.7 \%$ & \\
\hline
\end{tabular}

Fuente: Datos primarios encuesta a usuarios, Junio 2016

Este estudio ha reflejado que la mayoría de los usuarios no cuentan con una seguridad plena al abordar estas unidades, siendo expuestos a riesgos que puedan afectar en su seguridad sin embargo deben de agregar más unidades en horas picos para evitar la aglomeración de personas, para reducir el riesgo de la delincuencia se debe de contar con un personal capacitado a la hora de que se pueda presentar un acto de delincuencia con algún usuario, además deben cumplir con las leyes impuestas por el departamento de tránsito, para garantizar la seguridad que se brinda al viajar con las puertas cerradas.

\section{Estrategias que ayuden al mejoramiento del servicio del transporte urbano colectivo intramunicipal.}

\section{Línea Estratégica $N^{\circ}$ 1. Brindar seguridad de los usuarios.}

Objetivo: Proporcionar las condiciones básicas que exigen los parámetros de la ley 524 para la seguridad de los usuarios.

\section{Actividades:}

1. Evitar el exceso de pasajeros en las unidades.

2. Mantener cerradas las puertas de las unidades durante el recorrido.

3. Evitar frenasos por parte de los conductores de las unidades.

4. Respetar las Leyes de Tránsito.

\section{Línea Estratégica $\mathrm{N}^{\circ} 2$. Cumplir con los requisitos que exige la Ley 524 en relación al ambiente e higiene}

Objetivo: Ofrecer las condiciones adecuadas para un ambiente agradable y limpio. 


\section{Actividades:}

1. Limpiar las unidades al finalizar cada recorrido.

2. Exigir que cada unidad cuente, por lo menos, con un depósito de basura.

3. Concientizar a los usuarios para que depositen la basura en los lugares proporcionados por la unidad (esto por parte de los cobradores).

\section{Línea Estratégica $N^{\circ}$ 3. Mejorar los elementos de calidad que necesitan los usuarios}

Objetivo: Cubrir las demandas básicas señaladas por los usuarios.

\section{Actividades:}

1. Ofrecer unidades de fácil acceso para los usuarios (con escalones apropiados para subir cómodamente)

2. Reparar los asientos de las unidades, puesto que una gran mayoría están en mal estado.

3. Moderar el uso de la radio, no exceder el volumen del mismo.

\section{Línea Estratégica $N^{\circ} 4$. Brindar capacitaciones de relaciones humanas, técnicas de servicio al usuario por las autoridades pertinentes.}

Objetivo: Desarrollar las capacidades técnicas y humanas de los colaboradores

\section{Actividades:}

1. Capacitar con regularidad a los colaboradores en el área técnica del servicio prestado.

2. Exigir la participación de todos los colaboradores en charlas o cursos brindados por los entes reguladores.

3. Instruir a los colaboradores en temáticas de servicio al cliente y relaciones humanas.

4. Proporcionar un certificado por estar listo para laborar en una unidad de servicio de transporte.

\section{Línea Estratégica $N^{\circ}$ 5. Proporcionar nuevas unidades en la Ciudad de Estelí.}

Objetivo: Realizar un estudio para determinar el incremento de nuevas rutas.

\section{Actividades:}

1. Realizar una reunión con los propietarios para evaluar el uso de más unidades.

2. Aumentar el número de unidades especialmente en horas de mayor afluencia.

3. Contar con unidades de mayor capacidad de personas en horarios más demandados.

\section{Línea Estratégica $N^{\circ}$ 6. Implementar elementos innovadores para mejorar la calidad del servicio de transporte.}

Objetivo: Cubrir con las necesidades de los usuarios.

\section{Actividades:}

1. Proporcionar las condiciones adecuadas para personas discapacitadas.

2. Incluir una tercera puerta (salida de emergencia).

3. Implementar un sistema de cobro electrónico.

4. Regular el sonido de los radios en las unidades de transporte.

\section{CONCLUSIONES}

ElServiciodetransporteurbanocolectivointramunicipal de la ciudad de Estelí, implementa buenas estrategias en cuanto a la atención del servicio de transporte a los usuarios, en el estudio se logró observar y cuantificar mediante los instrumentos implementados en las personas encuestadas lo siguiente:

- Los usuarios encuestados están de acuerdo en función a la calidad del servicio, tomando en consideración que se encuestaron un total de 360 personas en las diferentes rutas de la Ciudad de Estelí.

- Los conductores encuestados aseguran que brindan 
un servicio (regular), tomando en cuenta que se aplicaron un total de 31 conductores y 58 ayudantes escogidos aleatoriamente en los diferentes puntos de la Ciudad de Estelí.

- Los usuarios han calificado la atención en un $51.4 \%$ como (regular) y un $27.2 \%$ como (muy buena) y el $21.4 \%$ lo consideran que la atención recibida es (mala), y con relación a la guía de observación aplicada a las unidades de transporte urbano colectivo intramunicipal se pudieron tener resultados negativos en cuanto a la atención brindada a los usuarios que utilizan el transporte, las condiciones físicas de la unidad varían en dependencia de las rutas de acuerdo a las unidades observadas los urbanos del sector del Barrio el Rosario son las que están mejores condiciones físicas, de atención, y de calidad.

- Muchos de los parámetros que se les pide a los propietarios de las unidades es que tenga una unidad segura, cómoda y limpia para los usuarios que utilizan el servicio, para brinda un servicio de calidad y que el usuario este satisfecho con estos parámetros.

- Una de los parámetros peor calificados por los usuarios es que las unidades en horas de mayor afluencia de personas se llenan completamente provocando en el usuario incomodad y otras situación de inseguridad en la integración física de estos.

Gracias a la realización efectiva de este estudio se pudo constatar que la hipótesis planteada en la investigación no se cumple por las razones siguientes:
- El 52.5\% de los usuarios encuestados calificaron que están satisfechos con la seguridad que brinda el servicio y $47.5 \%$ afirmo que no están satisfechos con la seguridad, en relación a la hipótesis planteada en la investigación este era uno de los indicadores a analizarse.

- El 53.3\% de los usuarios han estado de acuerdo con la presentación de los trabajadores de la unidad por tanto el 46.7 que no está de acuerdo.

- El 80\% dijo que si está de acuerdo con la trayectoria asignada a la ruta y el $20 \%$ restante no estaba de acuerdo con la trayectoria.

- $\quad$ El 73\% dijo que si estaba de acuerdo a la distancia que existía entre paradas y el $27 \%$ no estaba de acuerdo.

En conclusión los usuarios encuestados están de acuerdo con los parámetros establecidos en cuanto a satisfacción y relacionados con la hipótesis de la investigación.

\section{BIBLIOGRAFÍA}

Muñiz, G. (2011). Una definición de calidad. Zaragoza: Pearson Educacion.

Reyes, R. (31 de octubre de Octubre de 2011). Crece Negocios. Obtenido de http://www.crecenegocios. com/concepto-de-calidad/

Satillana, C. J. (2007). Conceptos basicos de transporte. Facua, 2.

Serna, H. (2006). De conceptos basicos de Servicio al cliente. Cartagena: Edicion Limitada. 